\title{
INFECTIOUS DISEASES, NSW: MARCH 2000
}

\section{TRENDS}

Notifications of infectious diseases through to January 2000 are shown in Table 1 and Figure 4. Notably, notifications of gonorrhoea appeared to have reached a plateau during 1999, after a steady rise since 1995. Other notifications appear to be in line with seasonal expectations.

\section{A CLUSTER OF LISTEROSIS IN THE HUNTER}

Listerosis is a food-borne disease caused by the bacterium Listeria monocytogenes. People who are immunocompromised, elderly and pregnant are particularly susceptible to the disease. ${ }^{1}$ The case-fatality rate for invasive disease is approximately 30 per cent ${ }^{1}$ and the incubation period ranges from three to 70 days. ${ }^{1}$

Food may become contaminated with Listeria, which are commonly found in the bowel of livestock, through crosscontamination in food processing facilities; or by contact with soil containing the bacterium. Because the contamination of raw foods is common, uncooked foods of animal origin (for example, meats and milk) and fruits and vegetables may present an infection risk to those who are susceptible. Infection can be prevented through:

- thorough cooking

- temperature control (Listeria can grow at $4^{\circ} \mathrm{C}$ )

- minimising storage time for high risk foods

- minimising cross contamination during the preparation of food

- $\quad$ avoiding high risk foods if susceptible. ${ }^{2}$

Nosocomial acquisition of listeriosis (for example, infection acquired by a person while in hospital by consuming contaminated food) has been recognised previously. ${ }^{2,3}$

The Hunter Public Health Unit usually receives between two and four notifications of cases of listeriosis each year. Between September 1997 and January 1999, nine cases of listeriosis were notified among Hunter residents. All of these individuals were either immunocompromised or elderly, and six died.

Because the first person to present in the cluster of cases had been a resident of a Hunter health care facility for 58 of the 70 days prior to the onset of their illness, the investigation of the cluster included assessment of the risk of nosocomial acquisition. Of all nine cases, six had been resident in Hunter health care facilities within the incubation period for listeriosis. Foods likely to have been consumed by the cases while they were in the health care facilities were sampled from the facilities' kitchens and tested for the presence of Listeria.

Listeria was isolated from the fruit salad supplied to the Hunter health care facilities by a local processor. Using molecular subtyping techniques this isolate was found to be a subtype indistinguishable from the clinical isolates from four of the cases (including three of the individuals who died). Relatives of three of the four cases infected with the same strain as that isolated from fruit salad could be contacted. One of the cases had a history of consumption of fruit salad while resident in Hunter health facilities. No history of consumption of fruit salad could be confirmed in the other two cases. While it is difficult to establish a causal link, in this cluster the similarity between the isolates found in the clinical specimens, and that found in the fruit salad, implicates the fruit salad as a likely source of infection.

All cases of listeriosis should be investigated to exclude possible nosocomial transmission. Isolates of Listeria obtained from infected individuals who have been resident in hospitals or aged care facilities should be submitted to the Institute of Clinical Pathology and Medical Research (ICPMR) laboratory at Westmead hospital for subtyping, to assist in the detection and investigation of clusters. The development of a central state and/or national database would provide information on the relative frequency of subtypes of isolates identified, and aid in the interpretation of epidemiological and environmental findings.

As residents of aged care and other health facilities are often immunocompromised, special measures are required to protect them from nosocomial listeriosis. The NSW Health Circular 99/95 describes measures to minimise the risk of infection with Listeria from a range of foods including fresh fruits and salad vegetables.

In response to the preliminary findings of this investigation, in late 1999 the NSW Chief Health Officer (CHO) wrote to all public and private hospitals advising of the potential risks associated with the consumption of fruit salad and other minimally processed foods. In addition, the $\mathrm{CHO}$ commissioned an expert group to review the implications of these findings for hospital food safety, and to develop appropriate recommendations. Further laboratory investigations related to this cluster of cases in the Hunter are under way, and a comprehensive report will be published on completion of the investigation.

\section{REFERENCES}

1. Benenson, AS (editor). Control of Communicable Disease Manual. Washington DC: American Public Health Association, 1995; 270-273.

2. Farber JM, Peterkin PI. Listeria monocytogenes, a foodborne pathogen. Microbiol Rev 1991; 55: 476-511.

3. Ho JL, Shands KN, Friedland G, Eckind P, Fraser DW. An outbreak of type $4 \mathrm{~b}$ Listeria monocytogenes infection involving patients from eight Boston hospitals. Arch Intern Med 1986; 146: 520-524. F 


\section{FIGURE 4}

REPORTS OF SELECTED INFECTIOUS DISEASES, NSW, JANUARY 1995 TO JANUARY 2000, BY MONTH OF ONSET

These are preliminary data: case counts in recent months may increase because of reporting delays
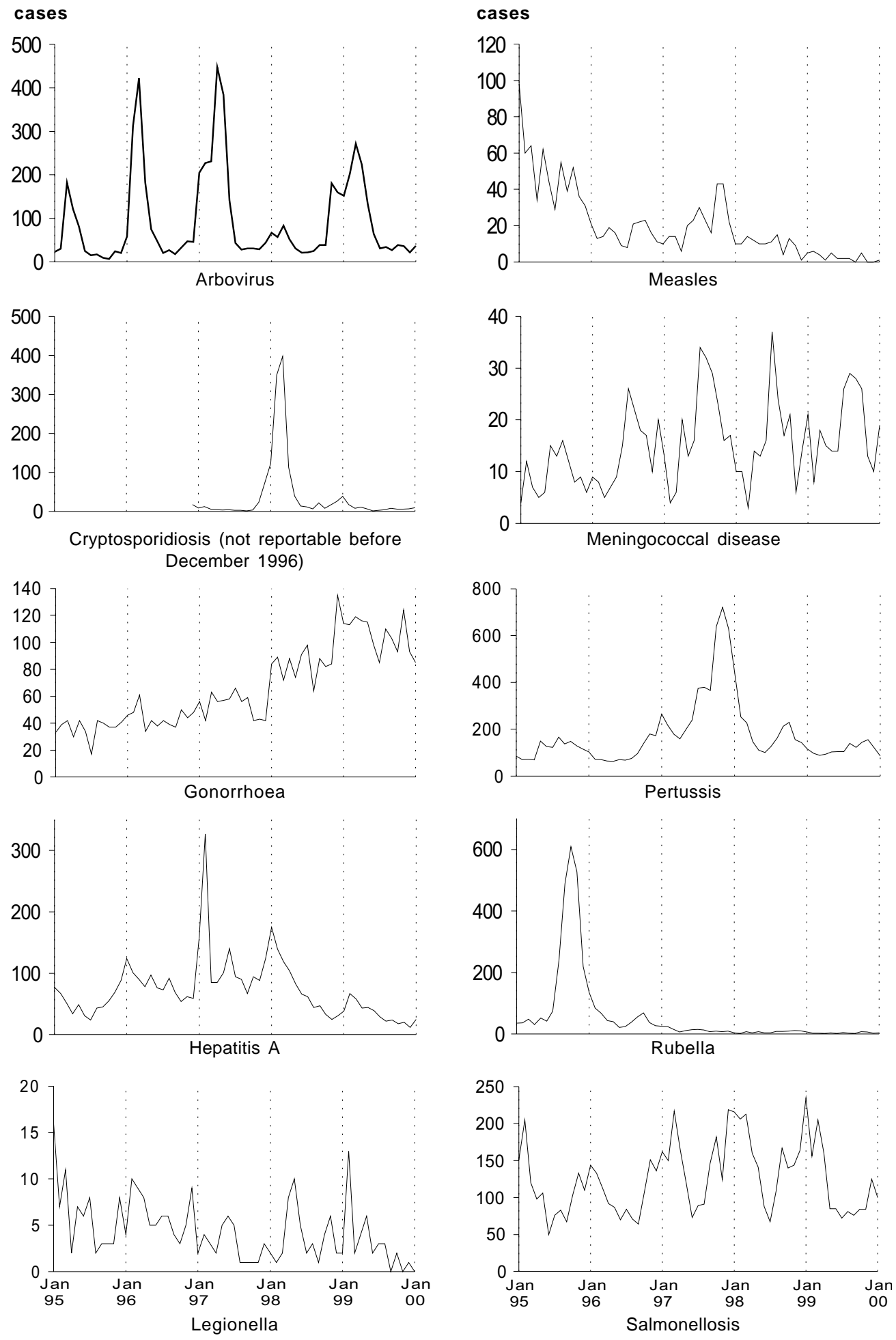


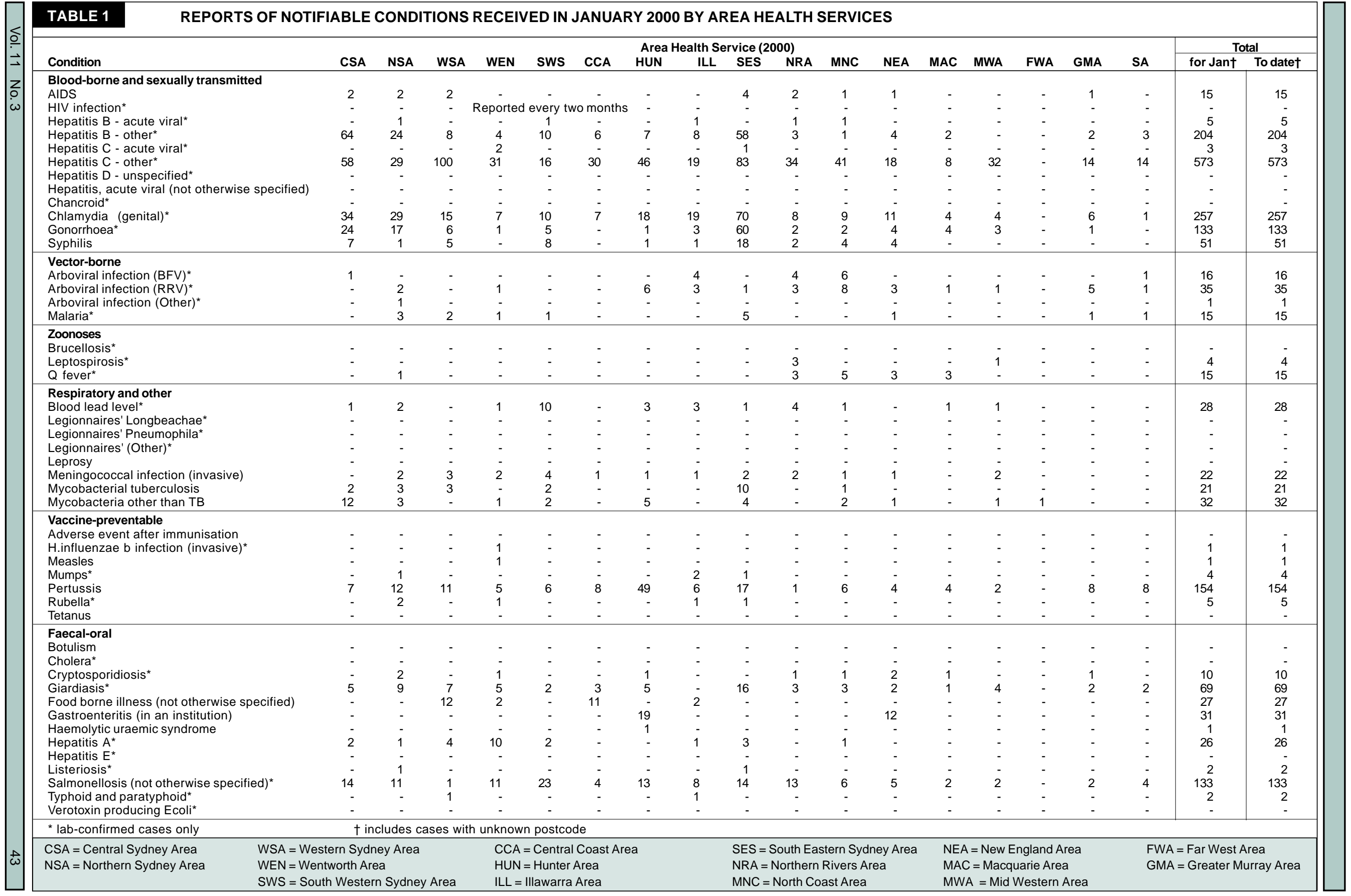

